\title{
Supporting Information \\ Energy-Efficient Hydrogen Evolution Reactions Via Hydrazine Oxidation Over Facile Synthesis of Cobalt Tetraoxide Electrodes
}

Xiaohu $\mathrm{Xu}^{\dagger}$, Tao Wang ${ }^{\dagger}$, Lijuan Dong $\ddagger$, Wenbo Lu ${ }^{\S}{ }^{*}$ and Xiangyang Miao ${ }^{* *}$

$\dagger$ Key Laboratory of Spectral Measurement and Analysis of Shanxi Province, College of Physics and Information Engineering, Shanxi Normal University, No.1 Gongyuan street, Yaodu District, Linfen 041004, China.

\Shanxi Provincial Key Laboratory of Microstructure Electromagnetic Functional Materials, Shanxi Datong University, Xingyun street, Nanjiao District Datong, 037009, China.

$\S$ Key Laboratory of Magnetic Molecules and Magnetic Information Materials (Ministry of Education), School of Chemistry and Material Science, Shanxi Normal University, No.1 Gongyuan street, Yaodu District, Linfen 041004, China.

*Corresponding author. E-mail: sxxymiao@126.com; Tel:+86-15035757788

*Corresponding author. E-mail: luwb@sxnu.edu.cn; Tel:+86-18935055015

Number of pages: 16

Number of figures: 13

Number of tables: 2 


\section{Content}

Experimental Section

SEM images for $10 \mathrm{~h}$ at $180{ }^{\circ} \mathrm{C}$

Fig. S1

LSV polarization curves, Nyquist plot and Plots of the $\mathrm{C}_{\mathrm{dl}}$

Fig. S2

SEM images for $20 \mathrm{~h}$ at $120^{\circ} \mathrm{C}$ and $150^{\circ} \mathrm{C}$

Fig. S3

XRD patterns for $20 \mathrm{~h}$ at $120{ }^{\circ} \mathrm{C}$ and $150{ }^{\circ} \mathrm{C}$ Fig. S4

LSV polarization curves, Nyquist plot and Plots of the $\mathrm{C}_{\mathrm{dl}}$ .Fig. S5

CV curves. Fig. S6

SEM images and XRD pattern .Fig. S7

XPS profiles .Fig. S8

LSV polarization curves and Nyquist plots Fig. S9

LSV polarization for synergetic effect. Fig. S10

Tafel plots Fig. S11

SEM images and XRD pattern after stability test Fig. S12

XPS profiles after stability test Fig. S13

Comparison of $\mathrm{HzOR}$ performance Table S1

Comparison of cell voltage. Table S2 


\section{Materials}

Acetone, ethanol, potassium hydroxide, hydrochloric acid, $\mathrm{Co}\left(\mathrm{NO}_{3}\right)_{2} \cdot 6 \mathrm{H}_{2} \mathrm{O}$ and hydrazine hydrate $\left(80 \mathrm{wt} \% \mathrm{H}_{4} \mathrm{~N}_{2} \cdot \mathrm{H}_{2} \mathrm{O}\right.$ ) were purchased from Aladdin. Both cobalt foam and nickel foam were purchased from Kunag Xun electronics Co., Ltd (KunShan, China). Pt/C (20 wt $\%$ ) and Nafion (5 wt \%) were obtained from Shanghai Macklin Biochemical Co., Ltd. All chemical regents were used as received without further purification. The deionized (DI) water was used during the experiments through a Millipore system.

\section{Characterization}

The X-ray powder diffraction (XRD) analysis was conducted through Bruker D8 Advance. The surface morphology of as-prepared samples were investigated by field-emission scanning electron microscopy (FE-SEM, Hitachi, S-4800), transmission electron microscopy (TEM, JEM-2100, $200 \mathrm{kV}$ ), and corresponding selected area electron diffraction (SAED, SU8010). The X-ray photoelectron spectroscopy (XPS) measurements were characterized with an X-ray photoelectron spectrometer (Thermo Scientific Escalab 250Xi), using Al as the exciting source.

\section{Synthesis of the $\mathrm{Pt} / \mathrm{C}$ catalyst on cobalt foam and $\mathrm{Co}_{3} \mathrm{O}_{4}$ catalyst onto glassy} carbon electrode (GCE)

The benchmark $\mathrm{Pt} / \mathrm{C}$ catalyst on a cobalt foam as the working electrode was prepared as the following steps: firstly, $13 \mathrm{mg}$ of $\mathrm{Pt} / \mathrm{C}$ was dispersed in $0.48 \mathrm{~mL}$ of ethanol with $0.02 \mathrm{ml}$ of Nafion solution. Secondly, 30 minutes sonication process was followed, and then $50 \mu \mathrm{L}$ of the catalyst suspension was dropped on the cobalt 
substrate with a geometrical surface area of $0.2 \mathrm{~cm}^{2}$. The loading amount is ca. $6.5 \mathrm{mg}$ $\mathrm{cm}^{-2}$. The process of $\mathrm{Co}_{3} \mathrm{O}_{4} / \mathrm{GCE}$ electrode preparation is as follows: firstly $4.55 \mathrm{mg}$ of $\mathrm{Co}_{3} \mathrm{O}_{4}$ catalyst powder was collected and dispersed in a mixture of ethanol $(45 \mu \mathrm{L})$ and 5 wt $\%$ Nafion solution $(5 \mu \mathrm{L})$, followed by slight ultrasonic process. Then $5 \mu \mathrm{L}$ of the catalyst ink was transferred onto GCE (diameter: $3 \mathrm{~mm}$, geometric area: 0.07 $\mathrm{cm}^{2}$ ) with the same loading mass of $6.5 \mathrm{mg} / \mathrm{cm}^{2}$ as $\mathrm{Co}_{3} \mathrm{O}_{4} / \mathrm{Co}$ and the as-prepared working electrode was obtained after dried at ambient temperature.

\section{Electrochemical measurements}

Cyclic voltammogram (CV), linear sweep voltammetry (LSV) polarization curves and electrochemical impedance spectroscopy (EIS) experiments were acquired by an electrochemical workstation (CHI 660E, Chenhua Instruments, China) with a three-electrode system, in which as-synthesized sample was used as working electrode, a saturated calomel electrode (SCE) as the reference one, and a carbon rod as the counter one, respectively. The potential region for $\mathrm{CV}$ is from -1.6 to $-0.6 \mathrm{~V}$ (vs. SCE) with a scan rate of $5 \mathrm{mV} \mathrm{s}^{-1}$. The frequency for EIS ranges from $100 \mathrm{KHz}$ to $1 \mathrm{~Hz}$ with an amplitude potential of $5 \mathrm{mV}$. The stability test was implemented using chronopotentiometric method $(\mathrm{j}-\mathrm{t})$ at certain potentials. All measured potentials (vs. SCE) here were converted to the reversible hydrogen electrode (RHE) according to the following formula: $\mathrm{E}(\mathrm{RHE})=\mathrm{E}(\mathrm{SCE})+0.0591 \times \mathrm{pH}+0.2415-0.000761(\mathrm{~T}-298.15)$ All the measurements above were corrected by manual iR compensation using the current and the solution resistance. 

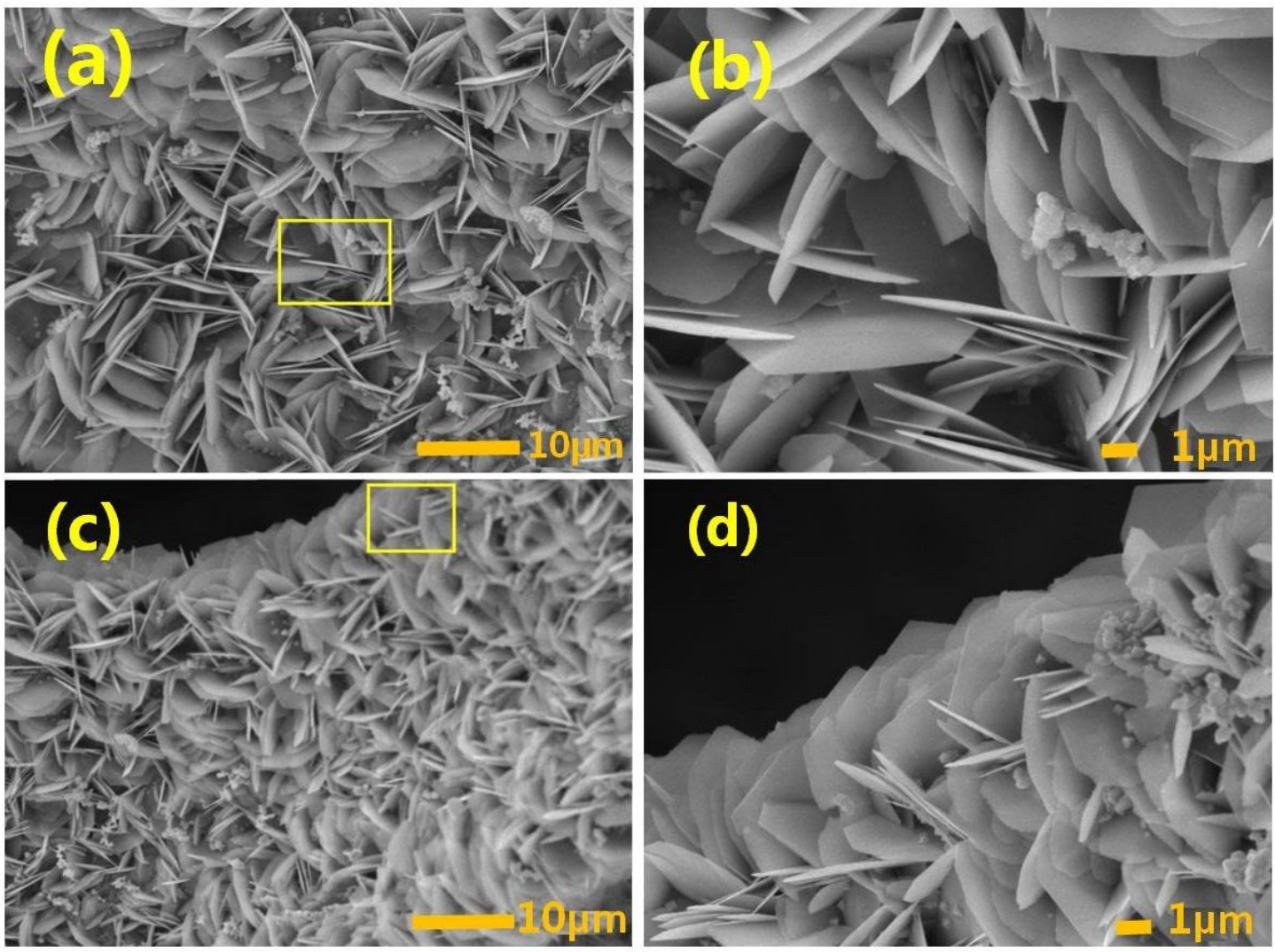

Fig. S1 SEM images of $\mathrm{Co}_{3} \mathrm{O}_{4} / \mathrm{Co}$ obtained by hydrothermal time for $10 \mathrm{~h}$ at $180{ }^{\circ} \mathrm{C}$.
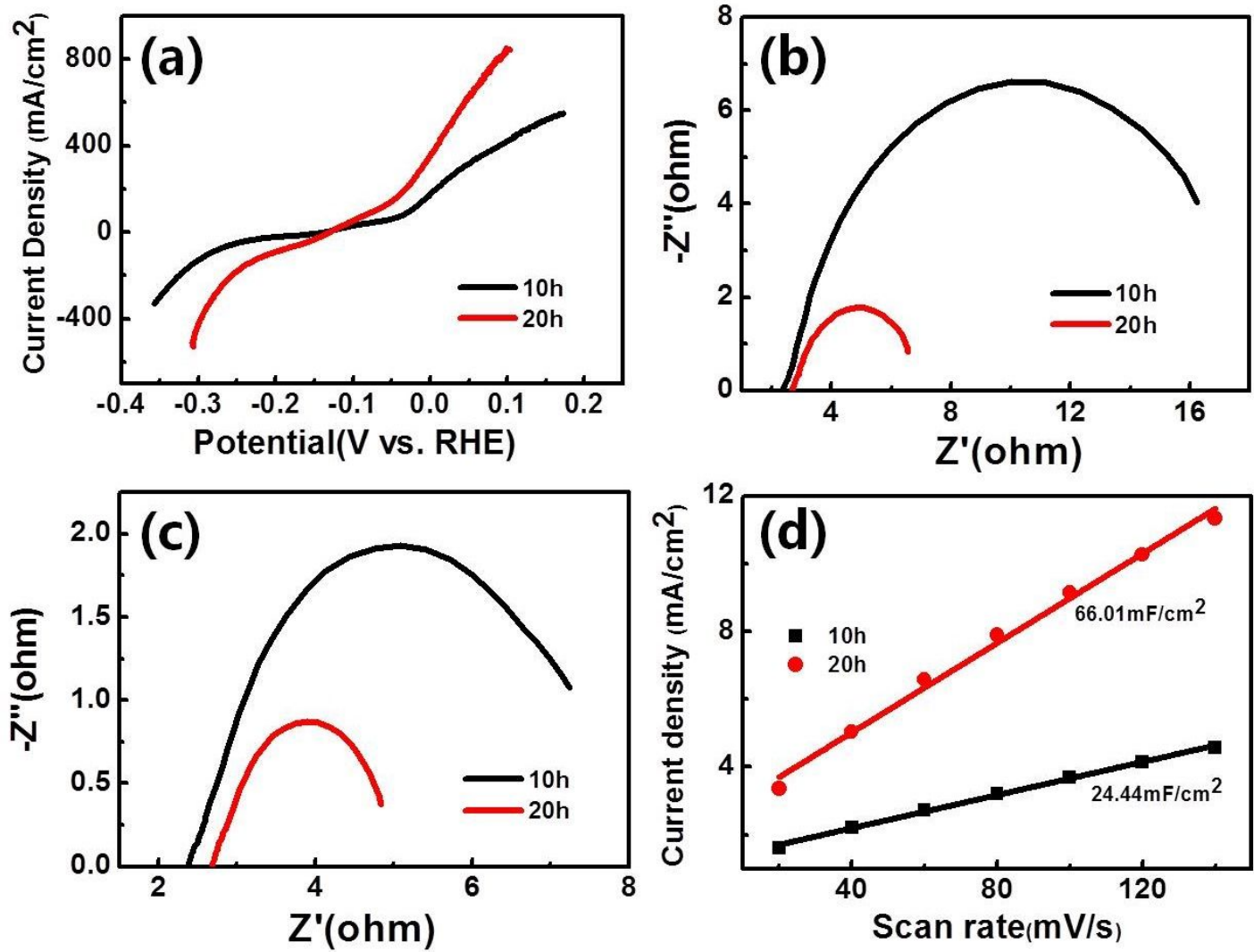

Fig. S2 (a) LSV polarization curves with iR compensation of $\mathrm{Co}_{3} \mathrm{O}_{4} / \mathrm{Co}$ with different 
hydrothermal time: 10 h, 20 h; (b), (c) Nyquist plot for HER and HzOR with different hydrothermal time in $1 \mathrm{M} \mathrm{KOH}$ with $0.3 \mathrm{M}$ hydrazine at $-200 \mathrm{mV}$ and $250 \mathrm{mV}$ vs RHE, respectively; (d) $\mathrm{C}_{\mathrm{dl}}$ of $\mathrm{Co}_{3} \mathrm{O}_{4} / \mathrm{Co}$ obtained from the data in $\mathrm{CV}$ curves recorded at different scan rate in $1.0 \mathrm{M} \mathrm{KOH}$ with different hydrothermal time.
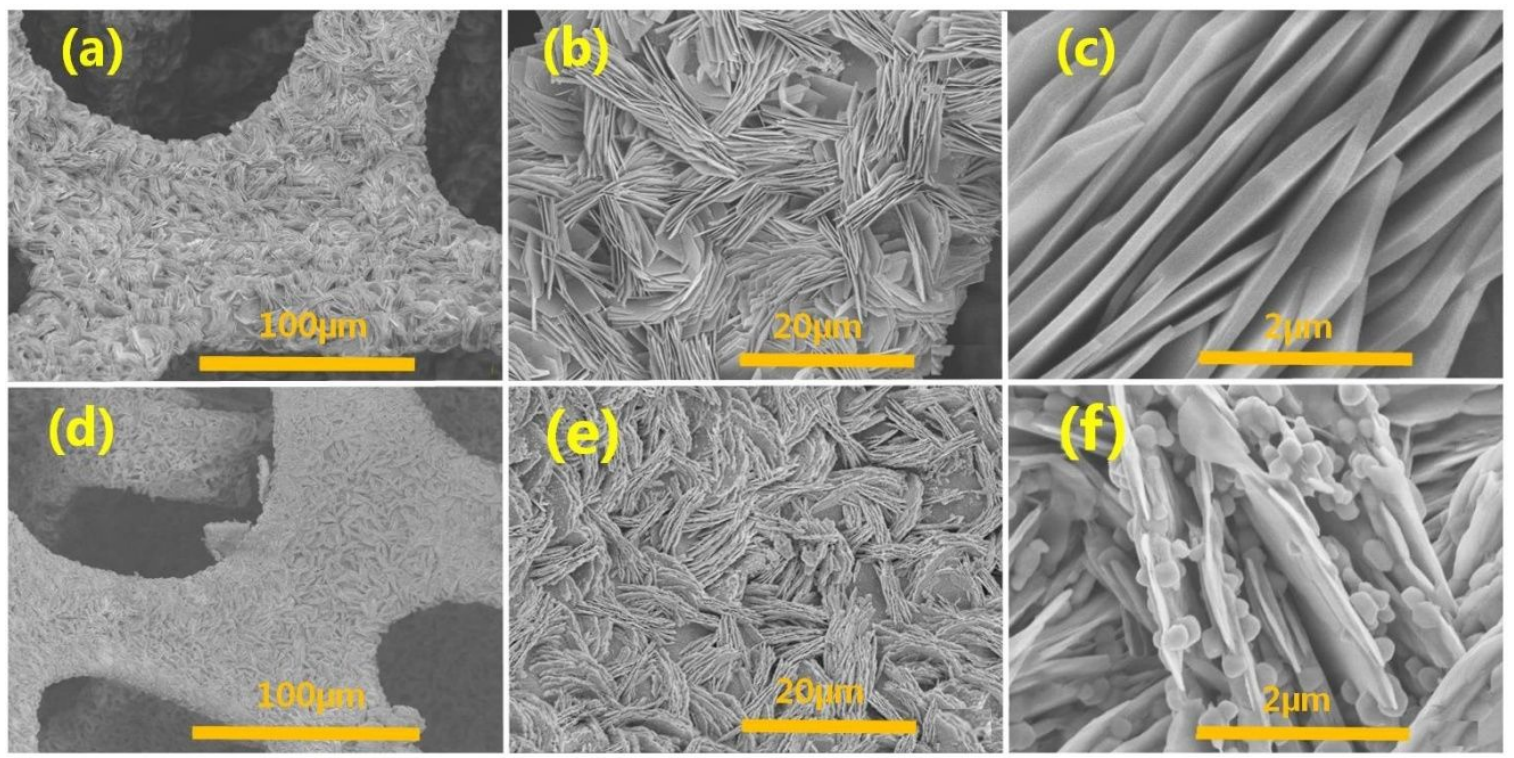

Fig. S3 SEM images of $\mathrm{Co}_{3} \mathrm{O}_{4} / \mathrm{Co}$ obtained by hydrothermal time for $20 \mathrm{~h}$ with different hydrothermal temperature: $(\mathrm{a}, \mathrm{b}, \mathrm{c}) 120^{\circ} \mathrm{C}$, (d, e, f) $150^{\circ} \mathrm{C}$.
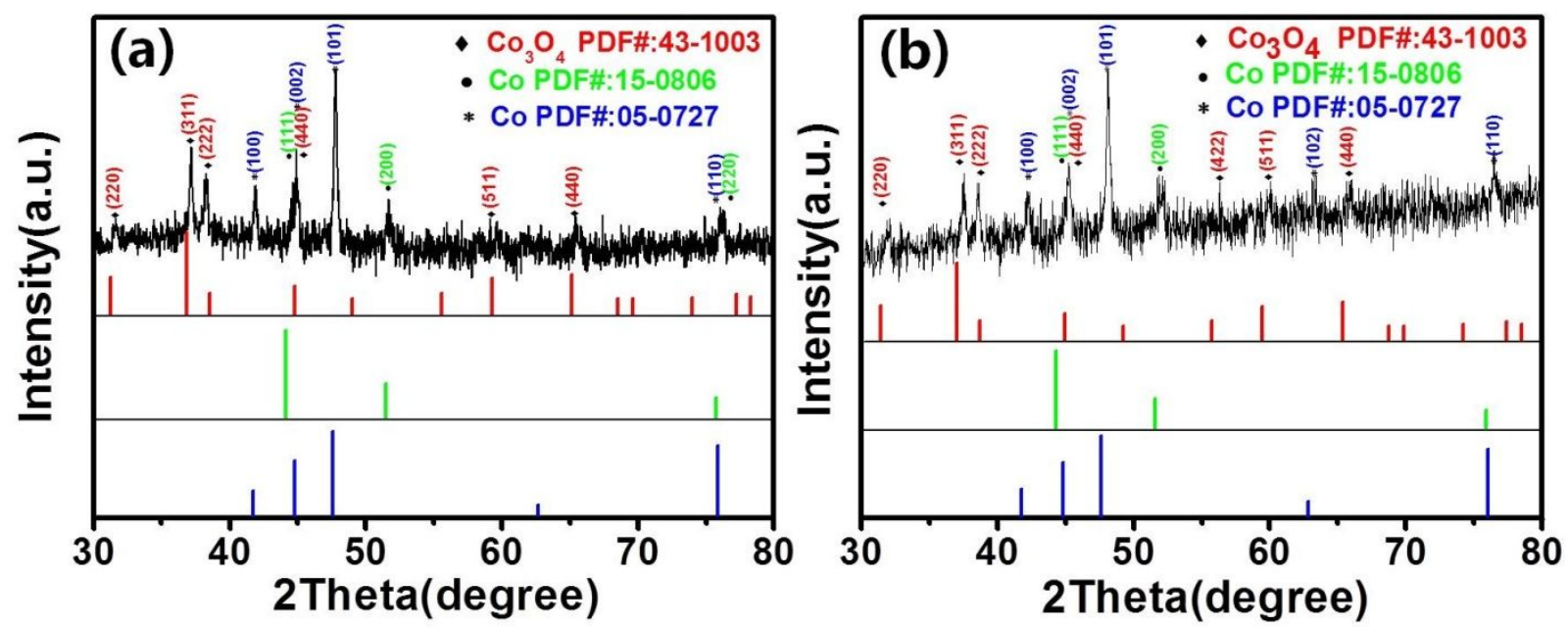

Fig. S4 XRD pattern of $\mathrm{Co}_{3} \mathrm{O}_{4} / \mathrm{Co}$ obtained by hydrothermal time for $20 \mathrm{~h}$ at different 
hydrothermal temperature: (a) $120^{\circ} \mathrm{C}$, (b) $150^{\circ} \mathrm{C}$.
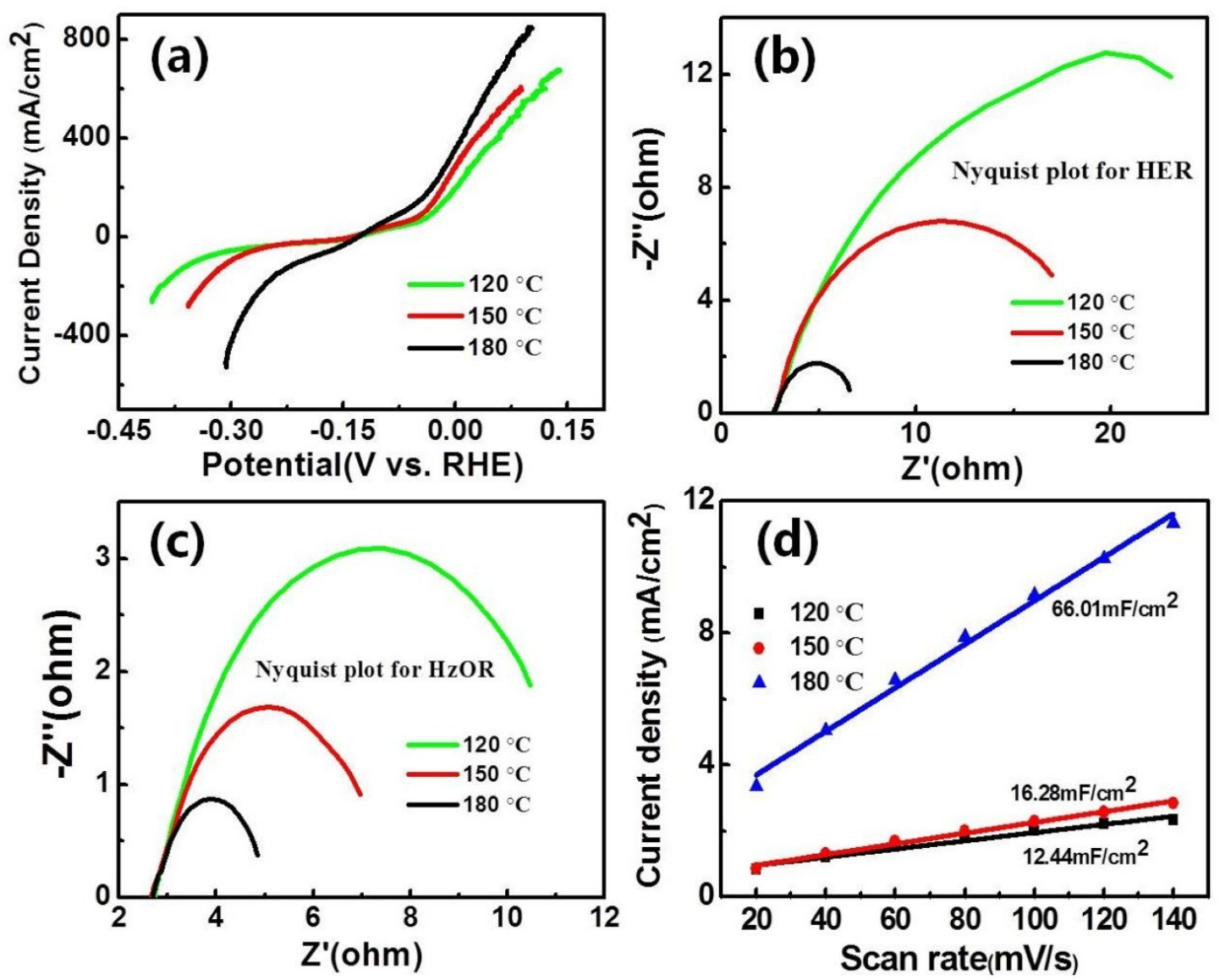

Fig. S5 (a) LSV polarization curves with iR compensation of $\mathrm{Co}_{3} \mathrm{O}_{4} / \mathrm{Co}$ at different hydrothermal temperature: $120^{\circ} \mathrm{C}, 150{ }^{\circ} \mathrm{C}, 180^{\circ} \mathrm{C}$; (b), (c) Nyquist plot for HER and $\mathrm{HzOR}$ with different hydrothermal temperature in $1 \mathrm{M} \mathrm{KOH}$ with $0.3 \mathrm{M}$ hydrazine at $-200 \mathrm{mV}$ and $250 \mathrm{mV}$ vs RHE, respectively; (d) $\mathrm{C}_{\mathrm{dl}}$ of $\mathrm{Co}_{3} \mathrm{O}_{4} / \mathrm{Co}$ obtained from the data in $\mathrm{CV}$ curves recorded at different scan rate in $1.0 \mathrm{M} \mathrm{KOH}$. 

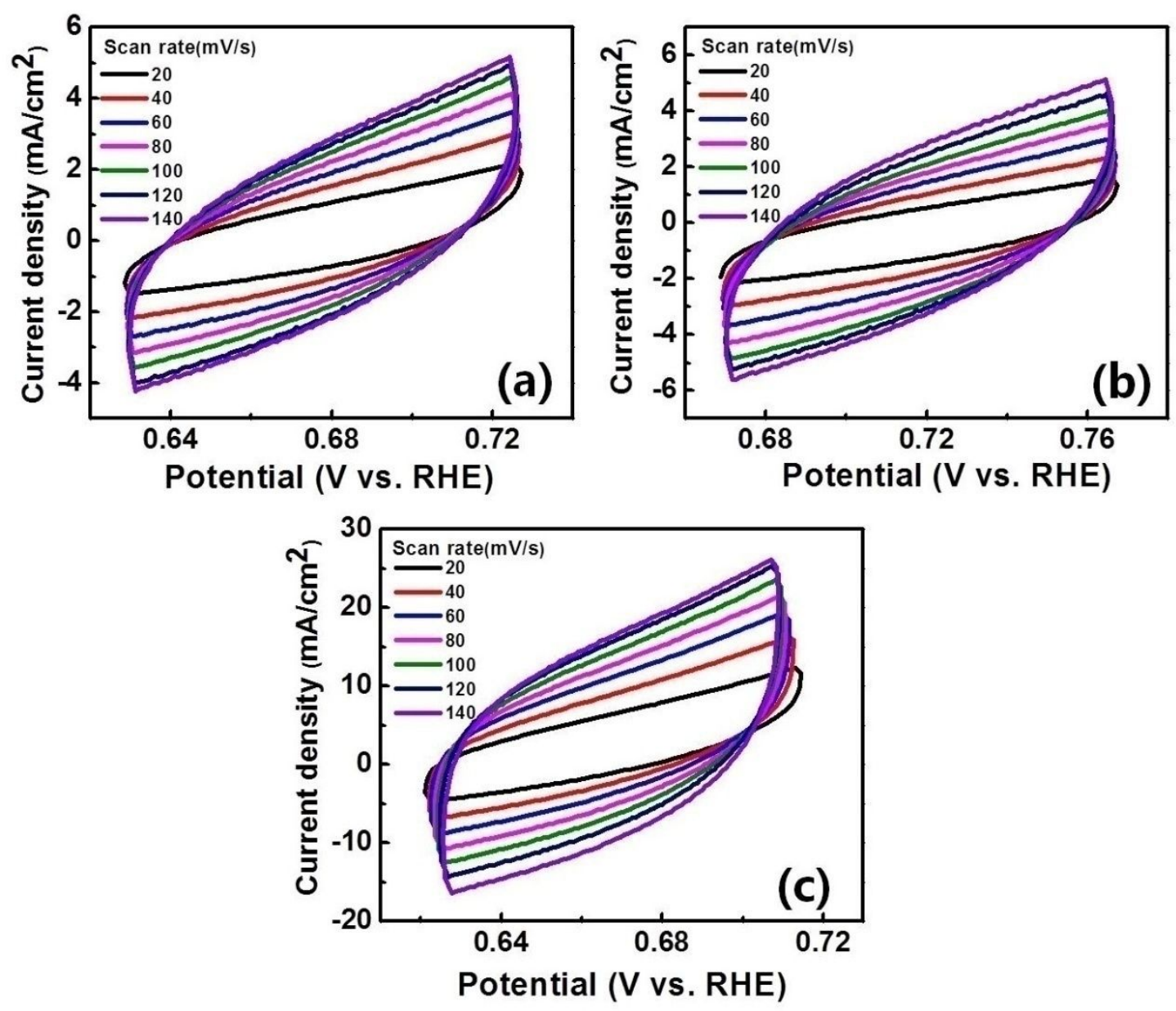

Fig. S6 Cyclic voltammetry of $\mathrm{Co}_{3} \mathrm{O}_{4} / \mathrm{Co}$ at different hydrothermal temperature: (a) $120{ }^{\circ} \mathrm{C}$, (b) $150{ }^{\circ} \mathrm{C}$ and (c) $180{ }^{\circ} \mathrm{C}$ from $20 \mathrm{mVs}^{-1}$ to $140 \mathrm{mVs}^{-1}$ in $1 \mathrm{M} \mathrm{KOH}$ in the nonfaradaic potential region. 

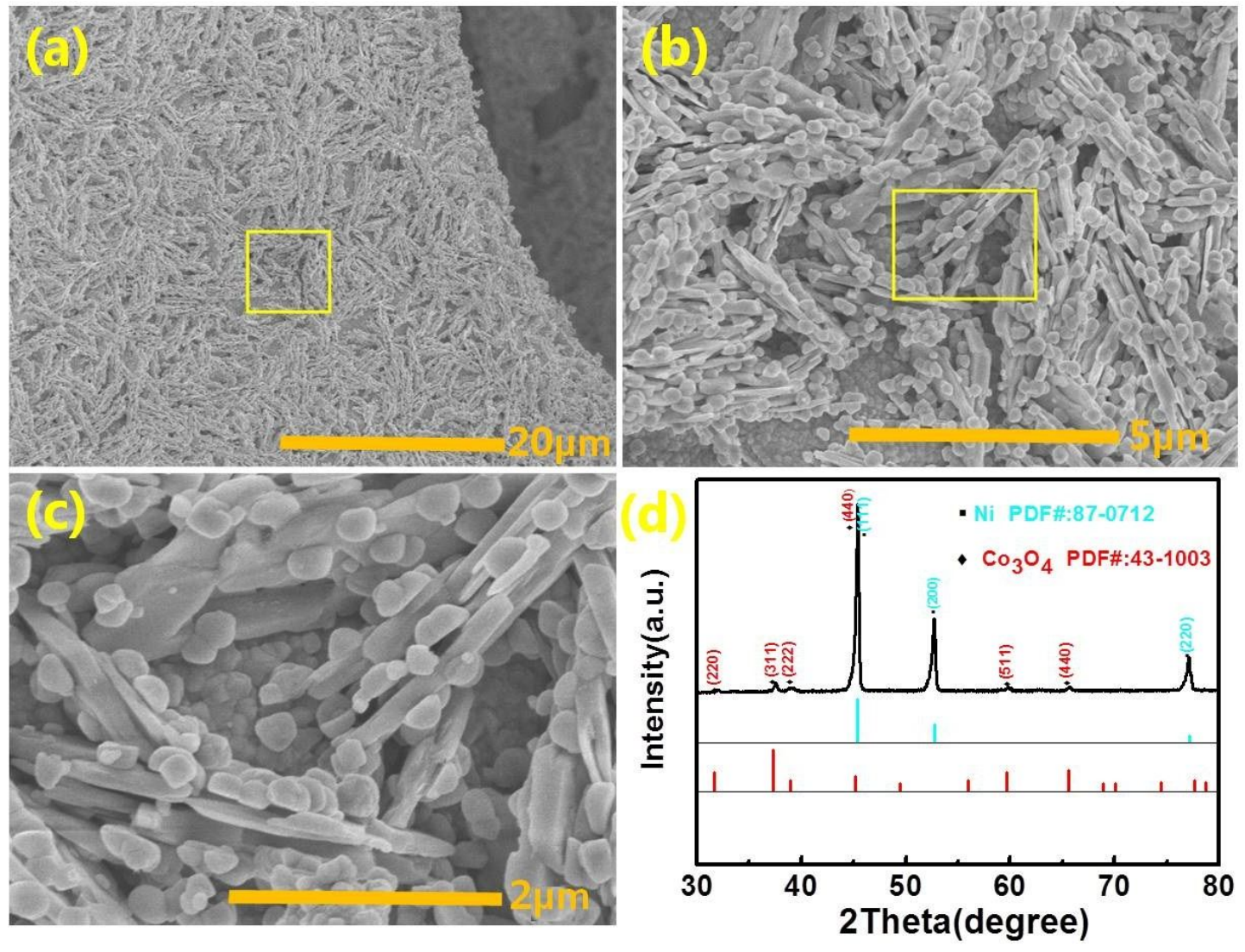

Fig. S7 ( $a, b$ and c) SEM images of $\mathrm{Co}_{3} \mathrm{O}_{4} / \mathrm{NF}$; (d) XRD patterns of $\mathrm{Co}_{3} \mathrm{O}_{4} / \mathrm{NF}$. 

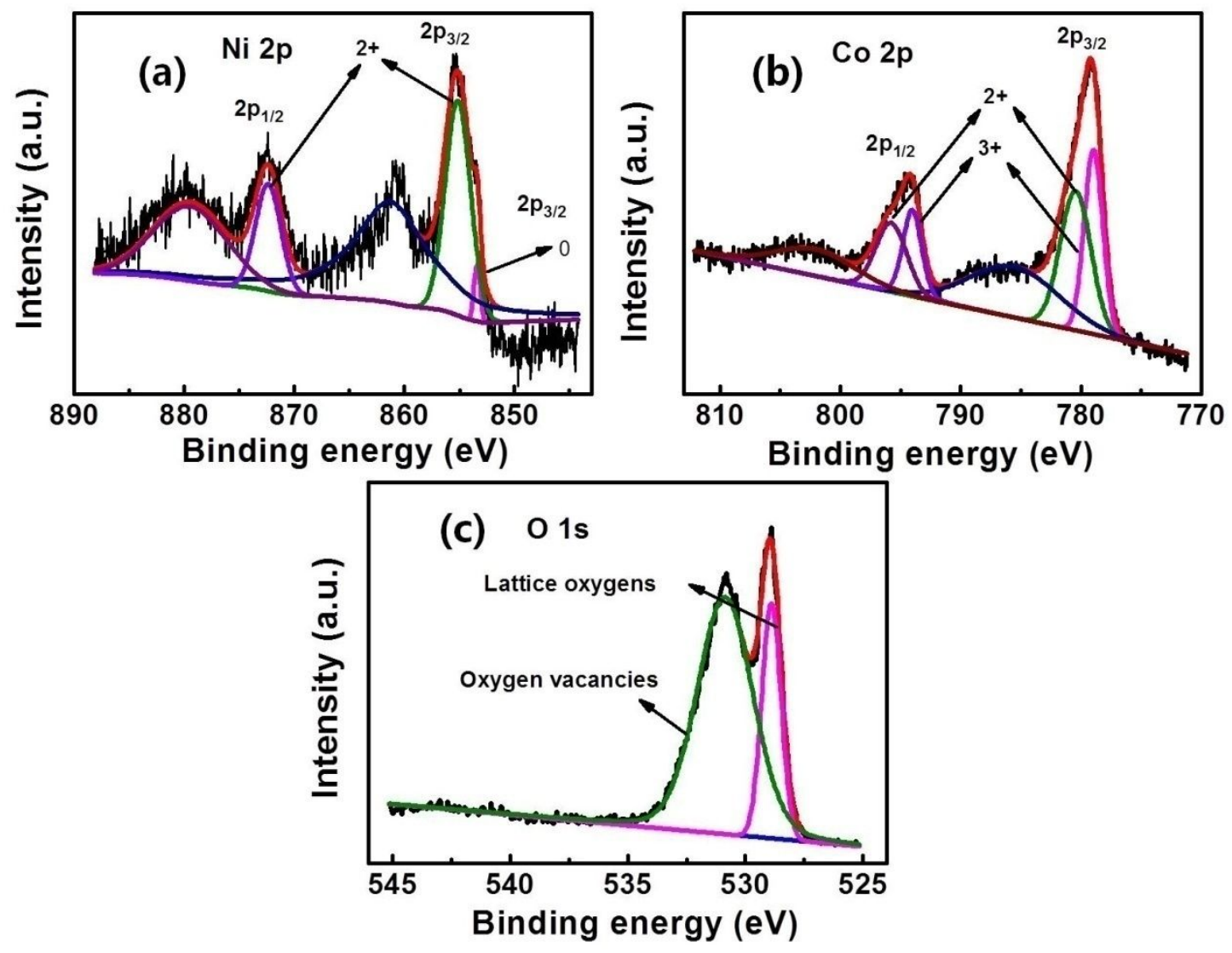

Fig. S8 XPS profiles of $\mathrm{Co}_{3} \mathrm{O}_{4} / \mathrm{NF}$ for (a) $\mathrm{Ni} 2 \mathrm{p}$, (b) Co $2 \mathrm{p}$ and (c) O 1 s. 

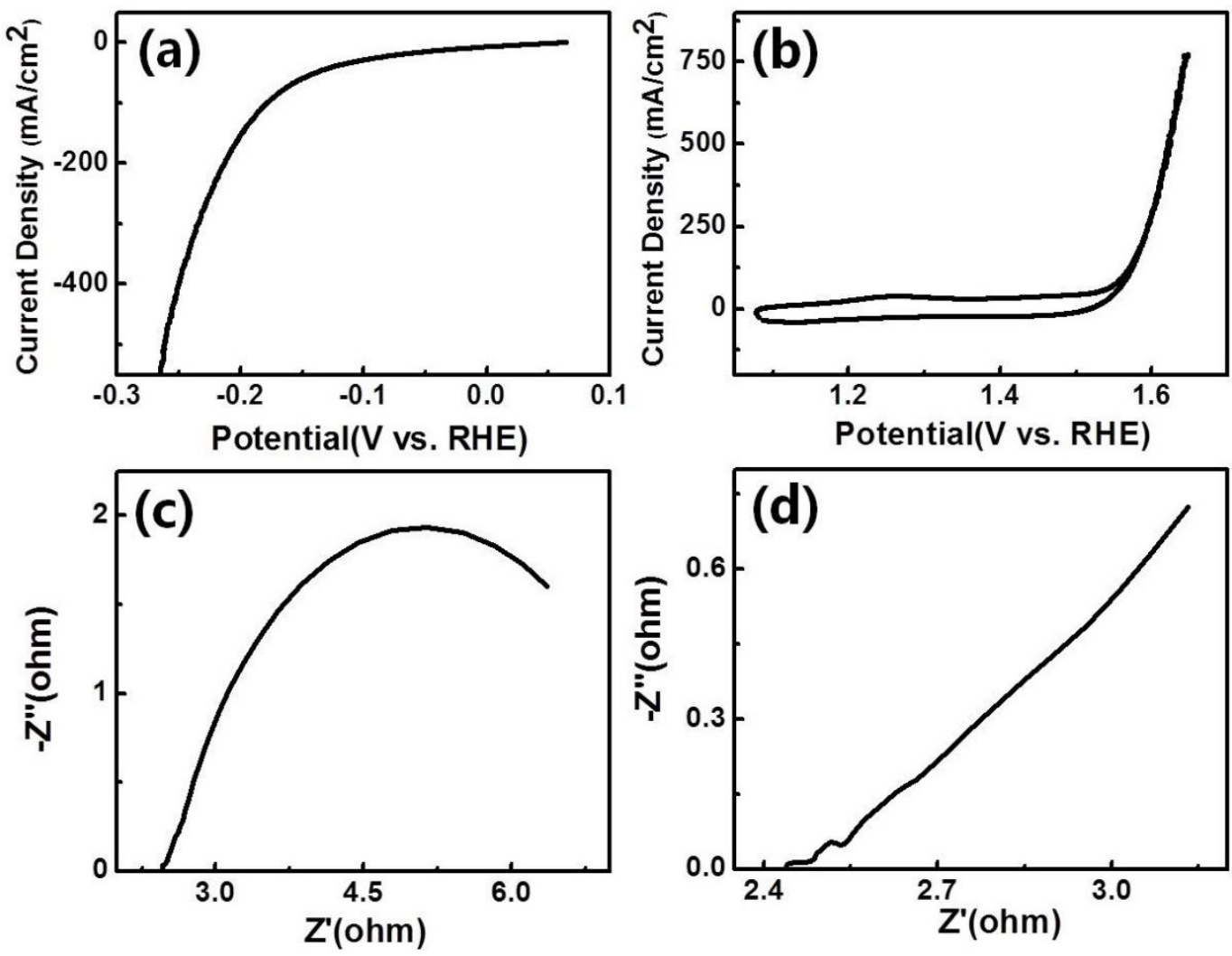

Fig. S9 LSV polarization curves with $\mathrm{iR}$ compensation of $\mathrm{Co}_{3} \mathrm{O}_{4} / \mathrm{Co}$ in $1 \mathrm{M} \mathrm{KOH}$ without hydrazine for (a) HER and (b) OER; (c), (d) Nyquist plot for HER and OER in $1 \mathrm{M} \mathrm{KOH}$ without hydrazine at-200 $\mathrm{mV}$ and $250 \mathrm{mV}$ vs RHE, respectively.

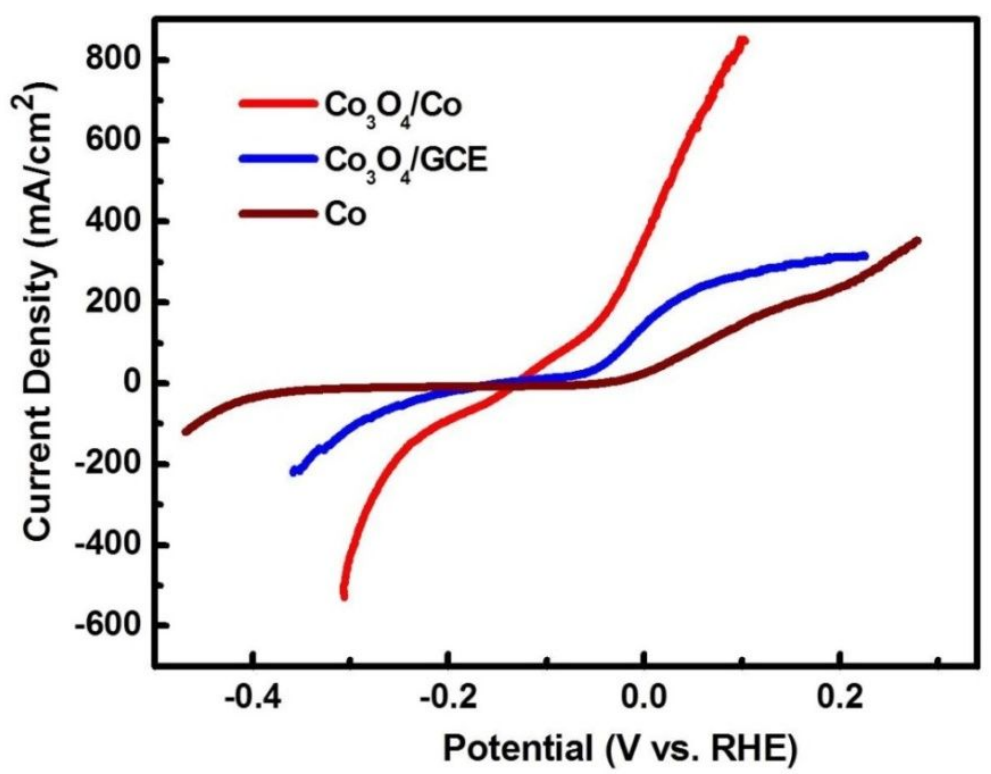

Fig. S10 LSV polarization curves with iR compensation of $\mathrm{Co}_{3} \mathrm{O}_{4} / \mathrm{Co}, \mathrm{Co}_{3} \mathrm{O}_{4} / \mathrm{GCE}$ and bare Co toward 0.3 M hydrazine. 

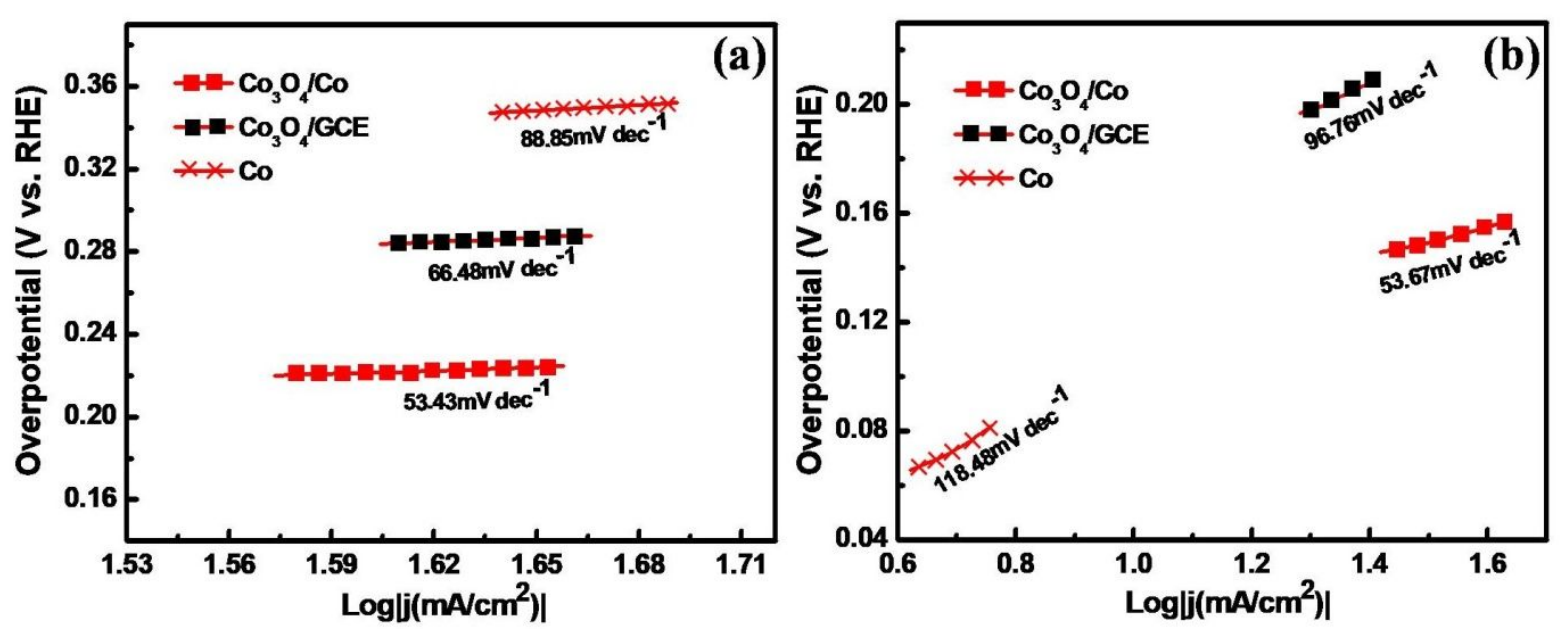

Fig. S11 Tafel plots for $\mathrm{HzOR}$ and HER of $\mathrm{Co}_{3} \mathrm{O}_{4} / \mathrm{Co}, \mathrm{Co}_{3} \mathrm{O}_{4} / \mathrm{GCE}$ and bare $\mathrm{Co}$

toward $0.3 \mathrm{M}$ hydrazine.
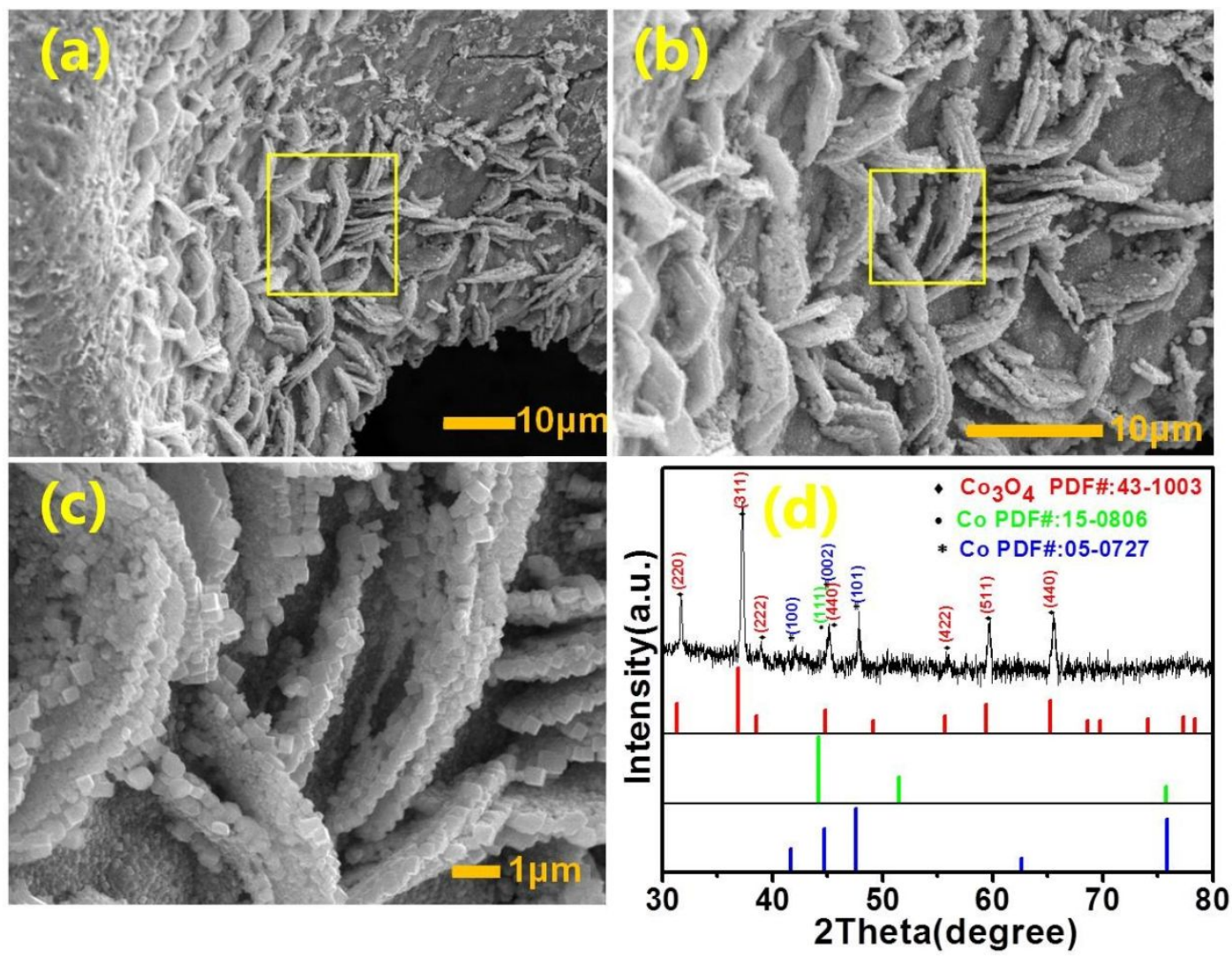

Fig. S12 (a, b and c) SEM images of $\mathrm{Co}_{3} \mathrm{O}_{4} / \mathrm{Co}$ after stability test; (d) XRD patterns of $\mathrm{Co}_{3} \mathrm{O}_{4} /$ Co after stability test. 

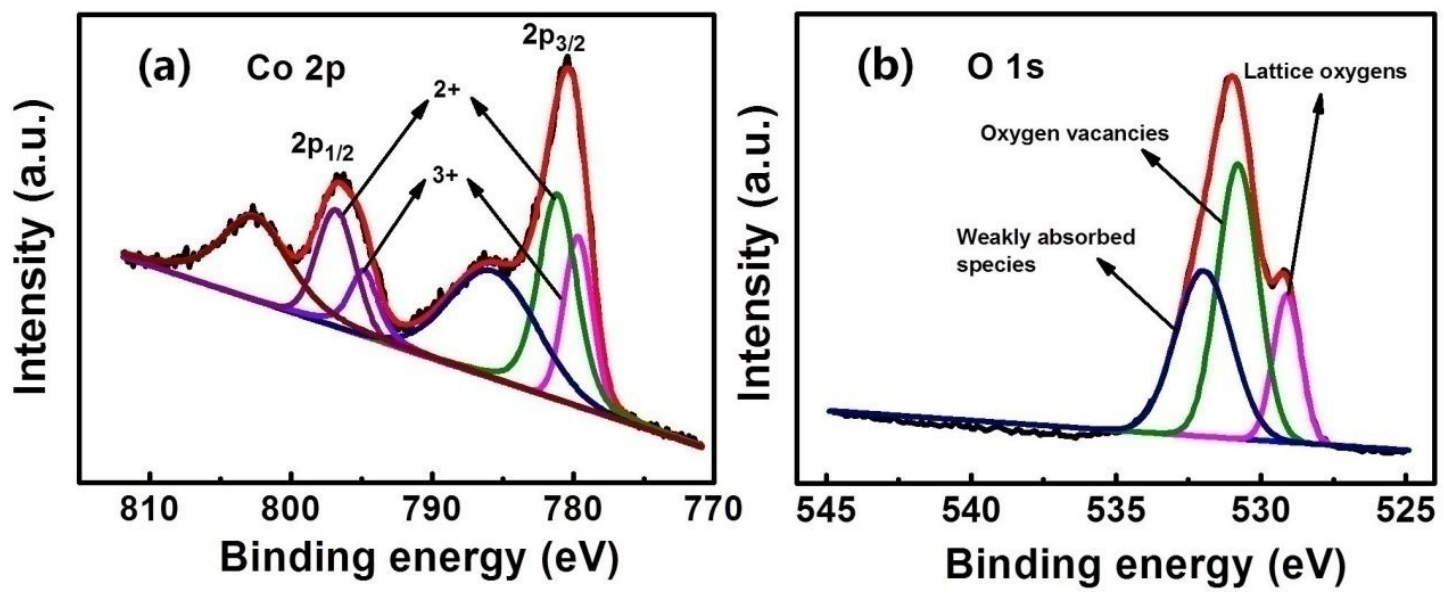

Fig. S13 XPS profiles of $\mathrm{Co}_{3} \mathrm{O}_{4} / \mathrm{Co}$ after stability test for (a) Co $2 p$ (b) O 1 s.

Table S1. Comparisons of $\mathrm{HzOR}$ catalytic activity of $\mathrm{Co}_{3} \mathrm{O}_{4} / \mathrm{Co}$ with the previous HzOR catalysts under alkaline solutions.

\begin{tabular}{|c|c|c|c|c|}
\hline Material & Electrolyte & $\begin{array}{l}\text { Tafel slope } \\
{\left[\mathrm{mVdec}^{-1}\right]} \\
\end{array}$ & Current density & Ref. \\
\hline $\mathrm{Co}_{3} \mathrm{O}_{4} / \mathrm{Co}$ & $\begin{array}{c}0.3 \mathrm{M} \mathrm{N}_{2} \mathrm{H}_{4} \\
1 \mathrm{M} \mathrm{KOH}\end{array}$ & 53.43 & $\begin{array}{c}50 \mathrm{mAcm}^{-2} \text { at }-0.11 \mathrm{~V} \\
\text { (vs. RHE) } \\
200 \mathrm{mAcm}^{-2} \text { at }-0.032 \mathrm{~V} \\
\text { (vs. RHE) } \\
300 \mathrm{mAcm}^{-2} \text { at }-0.014 \mathrm{~V} \\
\text { (vs. RHE) }\end{array}$ & This work \\
\hline $\mathrm{Ni}(\mathrm{Cu}) / \mathrm{NF}$ & $\begin{array}{c}0.5 \mathrm{M} \mathrm{N}_{2} \mathrm{H}_{4} \\
1 \mathrm{M} \mathrm{KOH}\end{array}$ & 51.2 & $\begin{array}{c}50 \mathrm{mAcm}^{-2} \text { at } 0.038 \mathrm{~V} \\
\text { (vs. RHE) } \\
100 \mathrm{mAcm}^{-2} \text { at } 0.086 \mathrm{~V} \\
\text { (vs. RHE) } \\
300 \mathrm{mAcm}^{-2} \text { at } 0.260 \mathrm{~V} \\
\text { (vs. RHE) }\end{array}$ & 1 \\
\hline $\mathrm{CoSe}_{2} / \mathrm{NF}$ & $\begin{array}{c}0.5 \mathrm{M} \mathrm{N}_{2} \mathrm{H}_{4} \\
1 \mathrm{M} \mathrm{KOH}\end{array}$ & - & $\begin{array}{c}100 \mathrm{mAcm}^{-2} \text { at } 0.17 \mathrm{~V} \\
\text { (vs. RHE) }\end{array}$ & 2 \\
\hline $\mathrm{Cu}_{3} \mathrm{P} / \mathrm{CF}$ & $\begin{array}{c}0.5 \mathrm{M} \mathrm{N}_{2} \mathrm{H}_{4} \\
1 \mathrm{M} \mathrm{KOH} \\
\end{array}$ & 60 & $\begin{array}{c}50 \mathrm{mAcm}^{-2} \text { at } 0.149 \mathrm{~V} \\
\text { (vs. RHE) }\end{array}$ & 3 \\
\hline
\end{tabular}




\begin{tabular}{|c|c|c|c|c|}
\hline $\mathrm{Cu}-\mathrm{Ni} / \mathrm{CuF}$ & $\begin{array}{c}3 \% \mathrm{~N}_{2} \mathrm{H}_{4} \\
5.5 \mathrm{M} \mathrm{KOH}\end{array}$ & - & $\begin{array}{c}14.3 \mathrm{mAcm}^{-2} \text { at }-0.58 \mathrm{~V} \\
\text { (vs. } \mathrm{Ag} / \mathrm{AgCl} \text { ) }\end{array}$ & 4 \\
\hline $\mathrm{NiCo}$ & $\begin{array}{c}0.1 \mathrm{M} \mathrm{N}_{2} \mathrm{H}_{4} \\
1 \mathrm{M} \mathrm{KOH}\end{array}$ & - & $\begin{array}{c}36 \mathrm{mAcm}^{-2} \text { at } 0.1 \mathrm{~V} \\
\text { (vs. RHE) }\end{array}$ & 5 \\
\hline $\begin{array}{c}\mathrm{Ni}_{80} \mathrm{Fe}_{20} \\
/ \mathrm{PEI}-\mathrm{rGO}_{10: 1}\end{array}$ & $\begin{array}{c}0.1 \mathrm{M} \mathrm{N}_{2} \mathrm{H}_{4} \\
0.15 \mathrm{M} \\
\mathrm{NaOH}\end{array}$ & - & $\begin{array}{c}57 \mathrm{mAcm}^{-2} \text { at } 0.5 \mathrm{~V} \\
\text { (vs. SCE) }\end{array}$ & 6 \\
\hline $\mathrm{NiS}_{2} / \mathrm{TiM}$ & $\begin{array}{c}0.5 \mathrm{M} \mathrm{N}_{2} \mathrm{H}_{4} \\
1 \mathrm{M} \mathrm{KOH}\end{array}$ & 22 & $\begin{array}{c}300 \mathrm{mAcm}^{-2} \text { at } 0.218 \mathrm{~V} \\
\text { (vs. RHE) }\end{array}$ & 7 \\
\hline $\mathrm{Ni}_{0.43} \mathrm{Cu}_{0.57} / \mathrm{Cu}$ & $\begin{array}{l}0.1 \mathrm{M} \mathrm{N}_{2} \mathrm{H}_{4} \\
3 \mathrm{M} \mathrm{KOH}\end{array}$ & - & $\begin{array}{c}300 \mathrm{mAcm}^{-2} \text { at }-0.6 \mathrm{~V} \\
\text { (vs. SCE) }\end{array}$ & 8 \\
\hline $\mathrm{Ni}_{0.6} \mathrm{Co}_{0.4}$ & $\begin{array}{c}0.5 \mathrm{M} \mathrm{N}_{2} \mathrm{H}_{4} \\
3 \mathrm{M} \mathrm{KOH}\end{array}$ & - & $\begin{array}{c}292 \mathrm{mAcm}^{-2} \text { at }-0.85 \mathrm{~V} \\
\text { (vs. SCE) }\end{array}$ & 9 \\
\hline $\mathrm{Ni}_{2} \mathrm{P} / \mathrm{NF}$ & $\begin{array}{c}0.5 \mathrm{M} \mathrm{N}_{2} \mathrm{H}_{4} \\
1 \mathrm{M} \mathrm{KOH}\end{array}$ & 55 & $\begin{array}{c}200 \mathrm{mAcm}^{-2} \text { at } 0.018 \mathrm{~V} \\
\text { (vs. RHE) }\end{array}$ & 10 \\
\hline Ni-NSA & $\begin{array}{c}0.5 \mathrm{M} \mathrm{N}_{2} \mathrm{H}_{4} \\
3 \mathrm{M} \mathrm{KOH}\end{array}$ & - & $\begin{array}{c}227.6 \mathrm{mAcm}^{-2} \text { at } 0.25 \mathrm{~V} \\
\text { (vs. RHE) }\end{array}$ & 11 \\
\hline 3D-PNNF & $\begin{array}{c}0.5 \mathrm{M} \mathrm{N}_{2} \mathrm{H}_{4} \\
3 \mathrm{M} \mathrm{KOH}\end{array}$ & - & $\begin{array}{c}198.6 \mathrm{mAcm}^{-2} \text { at } 0.25 \mathrm{~V} \\
\text { (vs. RHE) }\end{array}$ & 12 \\
\hline NPA-NiCuP & $\begin{array}{c}5 \mathrm{mM} \\
\mathrm{N}_{2} \mathrm{H}_{4}, 1 \mathrm{M} \\
\mathrm{KOH}\end{array}$ & - & $\begin{array}{c}51.44 \mathrm{mAcm}^{-2} \text { at } 0.36 \mathrm{~V} \\
\text { (vs. SCE) }\end{array}$ & 13 \\
\hline $\mathrm{NiB} / \mathrm{NF}$ & $\begin{array}{c}0.1 \mathrm{M} \mathrm{N}_{2} \mathrm{H}_{4} \\
1 \mathrm{M} \mathrm{NaOH}\end{array}$ & - & $\begin{array}{c}340 \mathrm{mAcm}^{-2} \text { at } 0.30 \mathrm{~V} \\
\text { (vs. RHE) }\end{array}$ & 14 \\
\hline $\mathrm{CoS}_{2} / \mathrm{TiM}$ & $\begin{array}{c}0.1 \mathrm{M} \mathrm{N}_{2} \mathrm{H}_{4} \\
1 \mathrm{M} \mathrm{KOH} \\
\end{array}$ & 48 & $\begin{array}{c}100 \mathrm{mAcm}^{-2} \text { at } 0.125 \mathrm{~V} \\
\text { (vs. RHE) }\end{array}$ & 15 \\
\hline
\end{tabular}


Table S2. Comparisons of cell voltage of noble-metal-free catalysts for $\mathrm{HzOR}$ and HER under alkaline solutions.

\begin{tabular}{|c|c|c|c|}
\hline Material & Electrolyte & Cell voltage & Ref. \\
\hline $\mathrm{Co}_{3} \mathrm{O}_{4} / \mathrm{Co} \| \mathrm{Co}_{3} \mathrm{O}_{4} / \mathrm{Co}$ & $\begin{array}{c}0.3 \mathrm{M} \mathrm{N}_{2} \mathrm{H}_{4} \\
1 \mathrm{M} \mathrm{КОН}\end{array}$ & $0.23 \mathrm{~V}$ for $100 \mathrm{mAcm}^{-2}$ & $\begin{array}{c}\text { This } \\
\text { work }\end{array}$ \\
\hline $\mathrm{Ni}(\mathrm{Cu}) / \mathrm{NF} \| \mathrm{Ni}(\mathrm{Cu}) / \mathrm{NF}$ & $\begin{array}{c}0.5 \mathrm{M} \mathrm{N}_{2} \mathrm{H}_{4} \\
1 \mathrm{M} \mathrm{KOH}\end{array}$ & $0.41 \mathrm{~V}$ for $100 \mathrm{mAcm}^{-2}$ & 1 \\
\hline $\mathrm{CoSe}_{2} / \mathrm{NF} \| \mathrm{CoSe}_{2} / \mathrm{NF}$ & $\begin{array}{c}0.5 \mathrm{M} \mathrm{N}_{2} \mathrm{H}_{4} \\
1 \mathrm{M} \mathrm{KOH}\end{array}$ & $0.164 \mathrm{~V}$ for $100 \mathrm{mAcm}^{-2}$ & 2 \\
\hline $\mathrm{Cu}_{3} \mathrm{P} / \mathrm{CF} \| \mathrm{Cu}_{3} \mathrm{P} / \mathrm{CF}$ & $\begin{array}{c}0.5 \mathrm{M} \mathrm{N}_{2} \mathrm{H}_{4} \\
1 \mathrm{M} \mathrm{KOH}\end{array}$ & $0.72 \mathrm{~V}$ for $100 \mathrm{mAcm}^{-2}$ & 3 \\
\hline $\mathrm{NiS}_{2} / \mathrm{TiM} \| \mathrm{NiS}_{2} / \mathrm{TiM}$ & $\begin{array}{c}0.5 \mathrm{M} \mathrm{N}_{2} \mathrm{H}_{4} \\
1 \mathrm{M} \mathrm{KOH}\end{array}$ & $0.75 \mathrm{~V}$ for $100 \mathrm{mAcm}^{-2}$ & 7 \\
\hline $\mathrm{Ni}_{2} \mathrm{P} / \mathrm{NF} \| \mathrm{Ni}_{2} \mathrm{P} / \mathrm{NF}$ & $\begin{array}{c}0.5 \mathrm{M} \mathrm{N}_{2} \mathrm{H}_{4} \\
1 \mathrm{M} \mathrm{KOH}\end{array}$ & $0.45 \mathrm{~V}$ for $100 \mathrm{mAcm}^{-2}$ & 10 \\
\hline $\mathrm{CoS}_{2} / \mathrm{TiM} \| \mathrm{CoS}_{2} / \mathrm{TiM}$ & $\begin{array}{c}0.1 \mathrm{M} \mathrm{N}_{2} \mathrm{H}_{4} \\
1 \mathrm{M} \mathrm{NaOH}\end{array}$ & $0.81 \mathrm{~V}$ for $100 \mathrm{mAcm}^{-2}$ & 15 \\
\hline
\end{tabular}

\section{REFERENCES}

[1] Sun, Q. Q.; Dong, Y. J.; Wang, Z. L.; Yin, S. W.; Zhao, C.; Bifunctional Copper-Doped Nickel Catalysts Enable Energy-Efficient Hydrogen Production via Hydrazine Oxidation and Hydrogen Evolution Reduction, ACS Sustainable Chem. Eng. 2018, 6, 12746-12754.

[2] Zhang, J. Y.; Wang, H. M.; Tian, Y. F.; Yan, Y.; Xue, Q.; He, T.; Liu, H. F.; Wang, C. D.; Chen, Y.; Xia, B. Y.; Anodic Hydrazine Oxidation Assists Energy-Efficient Hydrogen Evolution over a Bifunctional Cobalt Perselenide Nanosheet Electrode, Angew. Chem. Int. Ed. 57 (2018) 7649-7653.

[3] Liu, M.; Zhang, R.; Zhang, L. X.; Liu, D. N.; Hao, S. A.; Du, G.; Asiri, A. M.; Kong R.M.; Sun, X. P. Energy-efficient electrolytic hydrogen generation using a $\mathrm{Cu} 3 \mathrm{P}$ nanoarray as a bifunctional catalyst for hydrazine oxidation and water reduction. Inorg. Chem. Front., 2017, 4, 420-423.

[4] Filanovsky, B.; Granot, E.; Presmanb, I.; Kuras, I.; Patolsky, F. Long-term room-temperature hydrazine/air fuel cells based on low-cost nanotextured $\mathrm{Cu}-\mathrm{Ni}$ catalysts, J. Power Sources, 2014, 246, 423-429.

[5] Wang, H.; Ma, Y. J.; Wang, R. F.; Key, J. L.; Linkov, V.; Ji, S. Liquid-liquid interface-mediated room temperature synthesis of amorphous NiCo pompoms from ultrathin nanosheets with high catalytic activity for hydrazine oxidation, Chem. 
[6] Li, J.; Tang, W. J.; Yang, H. D.; Dong, Z. P.; Huang, J. W.; Li, S.W.; Wang, J.; Jin, J.; Ma,J. T. Enhanced-electrocatalytic activity of Nil-xFex alloy supported on polyethyleneimine functionalized $\mathrm{MoS}_{2}$ nanosheets for hydrazine oxidation, $R S C$ Adv.,

2014, 4, 1988-1995.

[7] Wang, J. M.; Ma, X.; Liu, T.Y.; Liu, D. N.; Hao, S. A.; Du, G.; Kong, R. M.; Asiri, A.M.; Sun, X.P. NiS2 nanosheet array: A high-active bifunctional electrocatalyst for

hydrazine oxidation and water reduction toward energy-efficient hydrogen production,

Materials Today Energy, 2017, 3, 9-14.

[8] Sun, M.; Lu, Z. Y.; Luo, L.; Chang, Z.; Sun, X. M. A 3D porous Ni-Cu alloy film for highperformance hydrazine electrooxidation. Nanoscale, 2016 , 8, 1479-1484.

[9] Feng, G.; Kuang, Y.; Li, P. S.; Han, N. N.; Sun, M.; Zhang, G. X.; Sun, X. M. Single crystalline ultrathin Nickel-Cobalt alloy nanosheets array for direct hydrazine fuel cells, $A d v$. Sci., 2017, 4, 1600179.

[10] Tang, C.; Zhang, R.; Lu, W. B.; Wang, Z.; Liu, D. N.; Hao, S. A.; Du, G.; Asiri, A. M.;Sun, X.P. Energy-saving electrolytic hydrogen generation: Ni2P nanoarray as a high-performance non-noble-metal electrocatalyst, Angew. Chem. Int. Ed. 2016, 55, $1-6$.

[11] Kuang, Y.; Feng, G.; Li, P. S.; Bi, Y. M.; Li, Y. P.; Sun, X.M. Single-crystalline ultrathin nickel nanosheets array from in situ topotactic reduction for active and stable electrocatalysis, Angew. Chem. Int. Ed. 2016, 55, 693-697.

[12] Feng, G.; Kuang, Y.; Li, Y. J.; Sun, X. M. Three-dimensional porous superaerophobic nickel nanoflower electrodes for high-performance hydrazine oxidation, Nano Res. 2015, 10, 3365-3371.

[13] Wang, X. L.; Zheng, Y. X.; Jia, M. L.; Yuan, L. S.; Peng, C.; Yang, W. H. Formation of nanoporous $\mathrm{NiCuP}$ amorphous alloy electrode by potentiostatic etching and its application for hydrazine oxidation. Int. J. Hydrogen Energy, 2016, 41, 8449-8458.

[14] Wen, X. P.; Dai, H. B.; Wu, L. S.; Wang, P. Electroless plating of Ni-B film as a binder-free highly efficient electrocatalyst for hydrazine oxidation, Appl. Surf. Sci. 2017,409, 132-139. 
[15] Ma, X.; Wang, J. M.; Liu, D. N.; Kong, R. M.; Hao, Sh.; Du, G.; Asiri, A. M. and Sun, X.P.; Hydrazine-assisted electrolytic hydrogen production: $\mathrm{CoS}_{2}$ nanoarray as a superior bifunctional electrocatalyst, New J. Chem., 2017, 41, 4754-4757. 